\title{
Diagnosing fungal infections in immunocompromised hosts
}

\author{
C M Tang, J Cohen
}

\section{Introduction}

Systemic fungal infections are increasing in incidence and importance, particularly in patients with haematological malignancies ${ }^{1}$ and in association with bone marrow and organ transplantation; $18-50 \%$ of patients develop an invasive fungal infection after bone marrow transplantation. ${ }^{23}$ Immunosuppressive treatment is also being used in an increasing number of conditions, such as colitis, nephritis, and asthma. Therefore patients with systemic mycoses are likely to present to doctors working in a wide range of specialties.

Mortality from systemic fungal infections in the immunocompromised remains depressingly high, in the order of $80 \% .^{1}$ The diagnosis is often only made at necropsy, not having been suspected clinically. Early treatment can reduce the mortality ${ }^{4}$ so it is extremely important to establish the diagnosis promptly.

The commonest pathogens in the United Kingdom are Candida spp, Aspergillus spp, and Cryptococcus neoformans. ${ }^{5}$ To make a definitive diagnosis of systemic infection with one of these organisms means that those responsible for the care of the patient have to have a high index of suspicion. In laboratories three general approaches are used.

Isolation of fungi from clinical specimens is not difficult, but does not necessarily indicate that they are pathogens. In some cases-the recovery of $C$ neoformans from cerebrospinal fluid for instance - the result is unambiguous, but in others-the isolation of $C$ albicans in the sputum-the interpretation depends entirely on the clinical setting. Hence laboratories should be wary of reporting fungal isolates as "normal flora" if the patient could be regarded as being "at risk" of invasive fungal disease. There is perhaps another general point that can be made here. Full identification of a fungal isolate, especially moulds, can take several days, time that is precious for the immunocompromised host. It is always worth giving the clinician preliminary information about potential pathogens, even if the result has to be amended later.

The second broad diagnostic approach available in the laboratory is the detection of an immune response to the organism (either antibody or antigen), or the measurement of some other marker of its presence, such as a metabolic product. The attraction of all these techniques is the potential speed with which they can be done, and the fact that they do not require "invasive" sampling procedures. In some cases this approach has been extremely useful-for example, the latex test for cryptococcal antigen-but it must always be remembered that few of these tests are sufficiently sensitive so as to exclude the diagnosis on the basis of a negative result. Antifungal treatment should never be withheld solely on the basis of a negative serological test.

Finally, fungal infection can often be best diagnosed by histological examination of an appropriate biopsy specimen. This need not entail undue delay; immediate examination of a "wet prep" or a simple smear stained with Giemsa can produce a diagnostic result within a very short time. Glycoproteins from fungal cell walls preferentially bind lectins ${ }^{6}$; if lectins are labelled with fluorescein, fungi in tissue sections could be rapidly identified.

There is no single "gold standard" for diagnosing deep fungal disease, a problem that has bedevilled the proper evaluation of fungal disease and its treatment. Each of the approaches we have described (and will discuss in more detail below) have their place, and always require close consultation between the clinician and the laboratory.

\section{Candidosis}

Most systemic candidal infections are caused by Candida albicans, though many other species of Candida, such as $C$ tropicalis, $C$ parapsilosis, $C$ glabrata, $C$ pseudotropicalis and $C$ krusei, may be pathogenic. ${ }^{7}$ Colonisation of the gastrointestinal tract with Candida spp occurs frequently, even in the non-immunocompromised host. This complicates the interpretation of clinical isolates. The clinical picture often provides few clues. Disseminated candidosis usually presents with a persistent fever and no diagnostic clinical features. ${ }^{8}$ Fundoscopy should be performed on all patients with suspected candidosis as candidal endophthalmitis, although only present in $5 \%$ of patients with confirmed invasive disease, is characteristic and its early recognition and treatment may save the patient's sight. ${ }^{9}$ Any nodular skin eruption of recent origin in a febrile, neutropenic patient should be biopsied as it may represent cutaneous emboli which are occasionally seen in patients with disseminated candidosis $^{10}$ and in some of the less common systemic mycoses.
Correspondence to: Dr J Cohen 
Blood cultures are part of the routine investigation of febrile neutropenic patients and in this setting isolates of Candida spp are highly predictive of invasive disease. Unfortunately Candida spp are grown from blood cultures in only $20 \%$ of those with definite disseminated candidosis. ${ }^{11}$ The yield can be improved using biphasic rather than vented blood culture media, ${ }^{12}$ while the lysis centrifugation system often gives an answer a day or two earlier than both these methods, especially in infections caused by $C$ tropicalis and $C$ glabrata. ${ }^{13}$ It is not practicable to use these alternative forms of blood cultures routinely, but they can be valuable in at-risk patients when a rapid diagnosis is important, or when other methods of culture have been unsuccessful. The finding of candiduria is harder to assess: many patients without systemic disease, and particularly those with indwelling urinary catheters, may have urinary tract colonisation with Candida spp. Nevertheless, interpretation of candiduria remains very difficult. It has been suggested that specimens containing more than $10^{4}$ organisms $/ \mathrm{ml}$ should be regarded as clinically significant, ${ }^{14}$ but this has never been properly validated. Similarly, the relevance of pyuria in association with candiduria has not been established; the absence of white cells is not helpful in patients who are neutropenic. Candiduria in immunosuppressed patients should always be reported by the laboratory, although it is not always an indication for systemic antifungal treatment.

An alternative approach to isolating the organism is to study the host's antibody response to the major cytoplasmic proteins of Candida spp. Serological studies used to involve identifying Candida precipitins in patients' serum; this proved extremely unreliable, with poor specificity and sensitivity. ${ }^{15}$ The main problem is that immunocompromised patients have poor antibody responses and when sensitive techniques, such as radioimmunoassay (RIA) and enzyme linked immunosorbent assay (ELISA), have been used an unacceptable number of false positive results are obtained in patients colonised with Candida spp. ${ }^{16}$ It has been suggested that the presence of circulating antibodies in a patient with invasive candidal disease is a good prognostic sign ${ }^{17}$; however, measurement of Candida antibodies remains of little help in establishing a diagnosis.

It had been hoped that finding circulating antigen may be a more accurate indicator of systemic disease. The major circulating antigen in candidosis is mannan, ${ }^{18}$ a heat stable, cell wall polysaccharide. It circulates in the form of immune complexes which are rapidly cleared by uptake in the Kuppfer cells of the liver and by glomerular filtration in the kidneys. ${ }^{19}$ Heat treatment of serum dissociates these complexes to liberate antigen for detection. Using RIA and ELISA, the specificity is high for disseminated candidosis but mannan is found in only $29-65 \%$ of patients. ${ }^{20}$ Immunoblot techniques permit the detailed study of circulating antigens and raise the possibility that antigens can be identified that are present only in invasive disease. ${ }^{22}$ One such antigen is a 48 kilodalton cytoplasmic protein which was found in the serum of $77 \%$ of affected patients. ${ }^{23}$

A much more rapid and convenient way of detecting circulating antigen is to use a latex agglutination test. Such a system has been developed $^{24}$ and is available commercially (Cand-Tec, Ramco Laboratories, Texas, USA). Latex particles are coated with serum from a rabbit previously immunised with heatkilled blastoconidia. The antigen detected by this method is a heat-labile glycoprotein that is not mannan. Using a cut-off titre of 1 in 8 as positive, the test is fairly specific (about $80 \%$ ) but not sufficiently sensitive, identifying antigenaemia in only $19-46 \%$ of immunosuppressed patients with disseminated disease. ${ }^{25} 26$ The sensitivity of the test is higher in those who are not immunosuppressed.

Candida spp produce a metabolite, Darabinitol, which can be detected using gasliquid chromatography (GLC). ${ }^{27}$ Unfortunately $\mathrm{D}$-arabinitol is found in low concentrations in some healthy people. Concentrations increase in renal impairment as it is cleared by the kidney at the same rate as creatinine; a Darabinitol : creatinine ratio can be calculated to compensate for this. This test is highly specific, but reported sensitivities vary from only 48$82 \%$. 2728

Most of the serological tests aimed at detecting antibody, antigen, or fungal metabolites are too insensitive to be of clinical use. The published sensitivity data can be improved by testing serial (often weekly) samples. This is of little benefit for the patient when an early diagnosis is required. Another problem is that most of the serological tests have been assessed in Candida albicans infections alone and will need to be evaluated for disease caused by the other species of Candida. The Cand-Tec test, however, detects antigenaemia in $C$ tropicalis and $C$ parapsilosis infections. ${ }^{24}$

\section{Aspergillosis}

Aspergillus spp are free-living saprophytes which are ubiquitous in the environment, present in the air as conidia. Aspergillus has been labelled the "weed of the culture room" as it often contaminates fungal culture plates. This highlights the difficulty of interpreting clinical isolates of Aspergillus, and having to decide if it is present as a contaminant, commensal, or pathogen. Infection is usually via inhalation of airborne spores (size $2-4 \mu \mathrm{m}$ in diameter) and is primarily pulmonary. In nonimmunocompromised hosts infection may elicit an allergic response, such as allergic bronchopulmonary aspergillosis, or be localised to a pre-existing lung cavity or damaged area of lung, producing a fungal ball or mycetoma. ${ }^{29}$ In immunocompromised patients the organism can proliferate in the alveoli, invade blood vessels, and disseminate haematogeneously, resulting in invasive aspergillosis. ${ }^{30}$ Despite this, blood cultures from patients with invasive aspergillosis are rarely positive. Other sites affected may include the paranasal sinuses, skin, central nervous system and the eye, causing endophthalmitis. ${ }^{31}$ As the 
lung is the main focus of infection, most efforts at making a diagnosis have been directed at examining respiratory samples.

Sputum culture for Aspergillus is of limited value, largely because the fungus may be found in the sputum of up to $15 \%$ of healthy people ${ }^{32}$ and because of the high numbers of negative results in patients with confirmed invasive aspergillosis (up to $70 \%)^{3334}$ A negative sputum result is therefore of little clinical importance and should not dissuade the clinician from making the diagnosis of invasive aspergillosis. On the othe hand, even a single sputum isolate, particularly of $A$ fumigatus or $A$ flavus in an immunocompromised patient, has been shown to be highly predictive of invasive aspergillosis. ${ }^{34} 35$

Of the more invasive ways of obtaining samples from the respiratory tract, open lung biopsy gives the highest diagnostic yield ${ }^{36}$ $(92 \%$ in patients with pulmonary infiltrates of unknown origin). Unfortunately patients are often too ill to undergo open biopsy and may have bleeding diatheses, complicating the procedure further. Most patients are able to tolerate fibre optic bronchoscopy; during the procedure bronchial washings, transbronchial biopsy, and bronchoalveolar lavage (BAL) can be performed to obtain samples. The isolation of Aspergillus spp from BAL fluid from immunosuppressed patients is highly indicative of invasive aspergillosis $\left(97 \%\right.$ specificity $\left.{ }^{37}\right)$ but is only positive in $50-58 \%$ of patients. ${ }^{37}{ }^{38} \mathrm{BAL}$ is the most helpful procedure for diagnosing invasive aspergillosis at bronchoscopy, comparing favourably with both bronchial washing (sensitivity $22 \%$ ) and transbronchial biopsy (sensitivity 18\%). Transbronchial biopsy specimens are often inadequate and obtained with increased risk, particularly in thrombocytopenic patients. Histological analysis must be combined with culture to make a definite diagnosis of invasive aspergillosis; a number of other fungi are indistinguishable from Aspergillus spp in tissue section (see below).

The major drawback of using bronchoscopically obtained specimens, apart from the lack of sensitivity, is that the diagnosis may be delayed. Bronchoscopy is usually undertaken after the patient has been treated with broad spectrum antibiotics for several days and Aspergillus may take a few days to grow in culture. As in candidosis, serological techniques have been applied to try and make a diagnosis sooner and by less invasive means. In allergic bronchopulmonary aspergillosis and in patients with mycetoma antibody responses are easily measured using either double diffusion or counterimmune electrophoresis. ${ }^{39}$ Not surprisingly, the case is different in the immunocompromised host who is unable to mount an adequate immune response and produce antibodies. Antibodies are unmeasurable in many patients, even when using sensitive techniques. ${ }^{17} 40$ Immunoblot techniques have identified an immunodominant 40 kilodalton antigen, though antibody to this was found in only $20 \%$ of patients with invasive aspergillosis. ${ }^{17}$
Because of disappointing results with antibody detection, interest has turned to the detection of circulating antigens. Galactomannan has been found in low concentrations in the serum of $66 \%$ patients with invasive aspergillosis when tested by RIA $^{41}$ and using ELISA. ${ }^{42}$ Galactomannan is a cell wall glycoprotein which binds lectin; $35 \%$ of an injected dose is found in urine over the following 24 hours. ${ }^{18}$ Antigen has been measured in urine and serum from all at-risk patients on a regular basis by both polyclonal and monoclonal ELISAs. ${ }^{43}$ Antigenaemia was detected before aspergillosis was clinically suspected in 16 out of 19 patients who subsequently developed invasive aspergillosis. ${ }^{43}$ In one instance antigen was detected 385 days before invasive aspergillosis was suspected; the relevence of such a result is not clear. While this approach seems attractive, weekly serum and urine testing of all immunocompromised patients is a large and expensive undertaking. A significant reduction in mortality from invasive aspergillosis would have to be shown before it could be recommended for widespread use.

High concentrations of D-mannitol, a fungal metabolite, have recently been found by GLC in the serum of rats with experimentally induced invasive aspergillosis. ${ }^{44}$ Clearly this has potential for use as a diagnostic marker for invasive aspergillosis in humans.

\section{Cryptococcosis}

The commonest clinical presentation of cryptococcosis is meningitis of gradual onset, usually with non-specific headaches, loss of memory, poor concentration and in some cases, signs of meningeal irritation and cranial nerve palsies. Papilloedema develops in $30 \%$ of patients and may be associated with a noncommunicating hydrocephalus. Cryptococcosis may also result in pneumonia or multiple skin nodules. The cerebrospinal fluid findings typically show a lymphocytosis, raised protein concentration, and a high blood : cerebrospinal glucose ratio. In immunocompromised hosts the course may be far more rapid and in some cases the cerebrospinal fluid results may be normal. ${ }^{45} 46$

India ink staining of the cerebrospinal fluid shows the typical yeasts of cryptococcosis in $50 \%$ of cases; the thick polysaccharide coat does not take up the stain and appears as a halo around the yeast. A latex agglutination test has been developed which detects capsular antigen. It is commercially available and is easy to use. It has a high sensitivity (95\%) for meningeal disease in both immunocompromised and nonimmunocompromised patients. False negative results can occur, more commonly in the nonimmunocompromised host (where the yeast load tends to be low) and when the organism is weakly encapsulated. It has been estimated that the lower limit of sensitivity for the detection of cryptococci in the cerebrospinal fluid by latex agglutination is $10^{3}$ organisms $/ \mathrm{ml}^{7}$ Therefore, cerebrospinal fluid should always be cultured and the clinician alerted immediately if a germ tube negative yeast is identified. If a rapid urease reaction is positive the likeliest 
aetiological cause is Cryptococcus neoformans. Rheumatoid factor can cause false positive results so the tests must have adequate controls. Capnocytophagia canimorsus (previously known as DF-2), ${ }^{48}$ malignancy, ${ }^{49}$ and systemic Trichosporon beigelii infection ${ }^{50}$ are rare causes of false positive results.

Not only is the latex test for cryptococcosis the most reliable of all the serological tests available for diagnosing systemic fungal infections, but serial titrations can be used as a useful guide to the response to treatment.

\section{Other fungal pathogens}

The list of potential fungal pathogens in immunocompromised patients is growing all the time. There is some evidence that infections caused by these unusual pathogens are becoming more common. ${ }^{51}$ They can be divided into (i) mucormycetes (or zygomycetes); (ii) dematiaceous fungi, which characteristically have septate hyphae and melanin in their cell walls; (iii) hyaline fungi; (iv) yeasts; and (v) others including Sporothrix schenkii and Pseudallescheria boydii. The interested reader is directed to some recent and comprehensive reviews. ${ }^{51-53}$

It is important to make an accurate diagnosis as not all of these fungi respond to standard treatment with amphotericin B. In many of these mycoses experience is limited and optimal treatment regimens remain unknown. In most instances the diagnosis can only be made definitely by culture as well as histology. This is because some of the dematiaceous and hyaline fungi appear identical to Aspergillus spp in tissue section unless special stains are used. Melanin is specifically stained by the Fontana Masson stain, so this can be used to differentiate dematiaceous fungi from Aspergilli and other hyaline fungi. ${ }^{51}$

The commonest clinical picture of infection with the mucormycetes is rhinocerebral mucormycosis. The usual causative agents are Rhizopus spp, Absidia spp, and Mucor spp. Infection begins in the paranasal sinuses and spreads, as a mass of ischaemic necrosis, through bone, to the orbit and frontal lobes. It is seen typically in patients with poorly controlled ketotic diabetes but is now occurring more frequently in neutropenic patients ${ }^{54}$ and those receiving haemodialysis. ${ }^{55}$ Mucormycetes may also cause pulmonary infection, multiple cutaneous lesions, and gastrointestinal ulcers. Diagnosis is confirmed by the demonstration of broad, widely branching, non-septate hyphae. It is not crucial to determine which species of mucormycete is responsible for the infection as they all respond similarly to amphotericin B. An ELISA was recently developed which detects antibody in patients' serum which reacted with antigen prepared from homogenates of $R$ arrhizus and $R$ pulsillus. ${ }^{56}$ In 43 patients with mucormycosis the test had a sensitivity of $81 \%$ with a $94 \%$ specificity. The patients were mainly diabetics with only two leukaemic patients tested, both of whom did not produce detectable antibody.

The hyaline fungi, including Fusarium spp, and the dematiaceous fungi, Bipolaris spp and Exserohilum spp, ${ }^{57}$ can all cause invasive disease that is clinically similar to aspergillosis. In fusariosis $70 \%$ of patients present with skin lesions, and blood cultures are positive in a high proportion of cases. Mortality in disseminated fusarial infection is of the order of $90 \%$ when treated with amphotericin $B$ alone $e^{58}$; in vitro the organism has reduced sensitivity to amphotericin $B$ and uniform resistance to 5flurocytosine..$^{59}$ Despite this, a combination of these two drugs has been used successfully in the treatment of fusarial infections. ${ }^{59}$

Several yeasts other than Candida spp can cause systemic infections. Trichosporon beigelii can produce disseminated infections which have a high mortality. Blood and urine cultures are frequently positive. Rhodoturula spp, Malassezia spp, and Saccharomyces spp may cause transient fungaemia in association with intravenous catheters. They may respond to the removal of the catheter alone.

The number of fungi that can cause systemic disease in the immunocompromised is now so great that isolates of all fungi from these patients must be viewed as being potentially pathogenic.

\section{Prospects}

Molecular biology is having an enormous impact in the diagnosis of many infectious diseases. Sequences of DNA which are specific for a given pathogen can be detected in clinical specimens by means of hybridisation to DNA probes, ${ }^{6061}$ strands of complementary DNA that can be labelled. It is now possible to amplify even a single strand of DNA to facilitate its detection using the polymerase chain reaction (PCR). The PCR is such a sensitive technique that it may be difficult to apply in infections where the aetiological organism can exist as a pathogen in one patient, and colonise another, as is the case in candidosis and aspergillosis. Patients colonised with the organism of interest, even in minute amounts, may give false positive results. Similar concerns about the use of the PCR in the diagnosis of pneumonia caused by Pneumocystis carinii, however, have proved to be unfounded. ${ }^{62}$ Studies are now underway to evaluate the PCR in fungal disease, and the results are awaited with interest.

It may be that with increasing prophylactic and early therapeutic use of amphotericin $B,{ }^{63}$ some of the less common fungi which are partially resistant to this drug will assume a more prominent place in the list of infectious complications confronting the immunocompromised patient. Already over the past 20 years the spectrum of infections has changed; the focus has shifted from bacterial to fungal pathogens. Similarly, as patients with AIDS live longer, invasive fungal diseases other than cryptococcosis, may become important rather than occasional pathogens. ${ }^{64}$

We thank the Leukaemia Research Fund and the British Society for Antimicrobial Therapy for financial support.

1 Gold JWM. Opportunistic fungal infections in patients with neoplastic disease. Am J Med 1984;76:458-63.

Pirsch JD, Maki DG. Infectious complications in adults with bone marrow transplantation and $\mathrm{T}$-cell depletion of with bone marrow transplantation and T-cell depletio 
3 Watson JG. Problems of infection after bone marrow transplantation. J Clin Pathol 1983;36:683-92.

4 Aisner J, Schimpff SC, Wiernik PH. Treatment of invasive aspergillosis: relation of early diagnosis and treatment to response. Ann Intern Med 1977;86:539-43.

5 Cohen J, Pinching AJ, Rees AJ, Peters DK. Infection and immunosuppression. A study of the infective complications of 75 patients with immunologically mediated disease. $Q J$ Med 1982;201:1-15.

6 Karayannopoulou G, Weiss J, Damjanov I. Detection of fungi in tissue sections by lectin histochemistry. Arch Pathol Lab Med 1987;112:746-8.

7 Stein DK, Sugar AM. Fungal infections in the immunocompromised host. Diagn Microbiol Infect Dis 1990;12: $221 \mathrm{~S}-8 \mathrm{~S}$.

8 Meunier F. Candidiasis. Eur J Clin Microbiol Infect Dis 1989;8:438-47.

9 Edwards JE, Foos RY, Montgonerie JZ, Guze LB. Ocular manifestations of Candida septicaemia: A review of 76 cases with haematogenous Candida endophthalmitis. Medicine 1974;53:47-75.

10 Balandrian L, Rothschild J, Pugh N, Seabury J. A cutaneous manifestation of systemic candidiasis. Ann Intern Med 1973;78:400-3.

11 Maksymiuk AW, Thongprasert S, Hopfer R, Luna M, Fainstein V. Fungal infections in cancer patients. Am J Fainstein V. Fung $1984 ; 77: 20-7$.

12 Kiehn TE, Capitolo C, Mayo JB, Armstrong D. Comparative recovery of fungi from biphasic and conventional blood culture media. J Clin Microbiol 1981;14:681-3.

13 Bille J, Edson RS, Roberts GD. Clinical evaluation of lysiscentrifugation blood culture system for the detection of fungemia and comparison with a conventional biphasic broth blood culture system. J Clin Microbiol 1984;19: 126-8.

14 Goldberg PK, Kozinn PJ, Wise GJ, Nouri N, Brooks RB. Incidence and significance of candiduria. JAMA 1979 241:582-4.

15 Filice G, Yu B, Armstrong D. Immunodiffusion and agglutination tests for Candida in patients with neoplastic disease: Inconsistent correlation of results with invasive disease: Inconsistent correlation of result

16 Lew MA. Diagnosis of systemic Candida infections. Ann Rev Med 1989;40:87-97.

17 Burnie JP, Matthews RC. Recent laboratory observations in the diagnosis of systemic fungal infection: Candida and Aspergillus. In: Holmberg, K, Meyer K, eds. Diagnosis and therapy of systemic fungal infections. New York: Raven Press Ltd, 1989:101-13.

18 Hopwood V, Warnock DW. New developments in the diagnosis of opportunistic fungal infection. Eur Microbiol Infect Dis 1986;5:379-88.

19 Bennett JE. Rapid diagnosis of candidiasis and aspergillosis. Rev Infect Dis 1987;9:398-402.

20 Bougnoux M-E, Hill C, Moissenet D, et al. Comparison of antibody, antigen and metabolite assays for hospitalized patients with disseminated or peripheral candidiasis. $J$ patients with disseminated
Clin Microbiol 1990;8:905-9.

21 de Repentigny L, Marr LD, Keller JW, et al. Comparison of enzyme immunoassay and gas-liquid chromatography for the rapid diagnosis of invasive candidiasis in cancer patients. J Clin Microbiol 1985;21:972-9.

22 Matthews RC, Burnie JP, Tabaqchali S. Isolation of immunodominant antigens from sera of patients with systemic candidiasis and characterization of serological response to Candida albicans. J Clin Microbio 1987;25:230-7.

23 Matthews R, Burnie J. Diagnosis of systemic candidiasis by enzyme-linked dot immunobinding assay for circulating immunodominant 47 kilodalton antigen. J Clin Microbiol immunodominant 4 .

24 Gentry LO, Wilkinson ID, Lea AS, Price MF. Latex agglutination test for detection of candida antigen in patients with disseminated disease. Eur J Clin Microbio 1983;2:122-8.

25 Cabezudo I, Pfaller M, Gerarden T. Value of the Cand-Tec candida antigen assay in the diagnosis and therapy of systemic candidiasis in high-risk patients. Eur J Microbio Infect Dis 1989;8:770-7.

26 Ness MJ, Vaughan WP, Woods GL. Candida antigen latex test for detection of invasive candidiasis in immunocompromised patients. J Infect Dis 1989;159:495-502.

27 Kiehn TE, Bernard EM, Gold JWM, Armstrong D. Candidiasis: detection by gas-liquid chromatography of $\mathrm{D}$ arabinitol, a fungal metabolite, in human serum. Science arabinitol, a fung
1979;206:577-80.

28 Gold JWM, Wong B, Bernard EM, Kiehn TE, Armstrong D. Serum arabinitol concentrations and arabinitol creatinine ratios in invasive candidiasis. J Infect Dis 1983;147:504-13.

29 Rippon JW. Medical mycology: pathogenic fungi and pathogenic actinomycetes. 3rd Edn. Philadelphia: WB Saunders Co, 1988.

30 Young RC, Bennett JE, Vogel CL, Carbone PP, DeVita VT. Aspergillosis; the spectrum of disease in 98 patients. Medicine 1970;49:147-73.

31 Cohen J. Clinical manifestations and management of aspergillosis in the compromised patient. In: Warnock DW, Richardson MD, eds. Fungal infection in the compromised patient. 2nd Edn. Chichester: John Wiley and Sons, 1991:117-52.

32 Warren RE, Warnock DW. Clinical manifestations and management of aspergillosis in the compromised host. In: the compromised patient. 1st Edn. Chichester: John Wiley the compromised patient.

33 Bodey GP. Fungal infections complicating acute leukaemia. JChron Dis 1966;19:667-87.

34 Yu VL, Muder RR, Poorsattar A. Significance of isolation of
Aspergillus from the respiratory tract in the diagnosis of invasive pulmonary aspergillosis. $\mathrm{Am} J \mathrm{Med}$ 1966;81: 249-54.

35 Nalesnick MA, Myerowitz RL, Jenkins R, Lenkey J, Herbert G. Significance of Aspergillus species isolated from respiratory secretions in the diagnosis of invasive pulmonary aspergillosis. J Clin Microbiol 1980;11:370-6.

36 Burt ME, Flye W, Webber BL, Welsey RA. Prospective evaluation of aspiration needle, transbronchial, and open lung biopsy in patients with pulmonary infiltrates. Ann Thorac Surg 1981;32:146-51.

37 Kahn FW, Jones JM, England DM. The role of bronchoalveolar lavage in the diagnosis of invasive pulmonary choalveolar lavage in the diagnosis of invasive pulm

38 Albelda SM, Talbot GH, Gerson SL, Miller WT, Cassileth PA. Role of fiberoptic bronchoscopy in the diagnosis of invasive pulmonary aspergillosis in patients with acute leukemia. Am J Med 1984;76:1027-34.

39 de Repentigny L. Serological techniques for diagnosis of fungal infection. Eur J Microbiol Infect Dis 1989;8:362-75.

40 Barnes RA. Immunological diagnosis of fungal infection in the immunocompromised host. Rev Med Microbiol 1990 1:58-65.

41 Talbot GH, Weiner MH, Gerson SL, Provencher $M$, Hurwitz S. Serodiagnosis of invasive aspergillosis in patients with hematologic malignancy: validation of the patients with hematologic malignancy: validation of the Dis 1987;155:12-27.

42 Sabetta JR, Miniter P, Andriole VT. The diagnosis of invasive aspergillosis by an enzyme-linked immunosorbent assay for circulating antigen. $J$ Infect Dis

43 Rogers TR, Haynes KA, Barnes RA. Value of antigen detection in predicting invasive pulmonary aspergillosis. Lancet 1990;336:1210-13.

44 Wong B, Brauer KL, Tsai RR, Jayasimhulu K. Increased amounts of the Aspergillus metabolite D-mannitol in tissue and serum of rats with experimental aspergillosis. $J$ Infect Dis 1989;160:95-103.

45 Dismukes WE. Cryptococcal meningitis in patients with AIDS. $J$ Infect Dis 1988;157:624-8.

46 Shaunak S, Schell WA, Perfect JR. Cryptococcal meningitis with normal cerebrospinal fluid. J Infect Dis 1989;160:912.

47 Cohen J. Comparison of the sensitivity of three methods for the rapid identification of Cryptococcus neoformans. $J$ Clin Pathol 1984;37:332-4.

48 Westernick MAL, Amsterdam D, Petell RJ, Stram MN, Apicella MA. Septicemia due to DF-2: Cause of falsepositive cryptococcal latex agglutination result. $\mathrm{Am} \mathrm{J} \mathrm{Med}$ 1987;83:155-8.

49 Hopfer RL, Perry EV, Fainstein V. Diagnostic value of cryptococcal antigen in the cerebrospinal fluid of patients with malignant disease. $J$ Infect $D$ is 1982;145:915

50 McManus EJ, Jones JM. Detection of Trichosporon beigelii antigen cross-reactive with Cryptococcus neoformans capsular polysaccharde in serum from a patient with disseminated Trichosporon infection. J Clin Microbiol disseminated 5 Tr:

51 Anaissie EJ, Bodey GP, Rinaldi MG. Emerging fungal pathogens. Eur J Microbiol Infect Dis 1989;8:323-30.

52 Skahan KJ, Wong B, Armstrong D. Clinical manifestation and management of mucormycosis in the compromised patient. In: Warnock DW, Richardson MD, eds. Fungal infection in the compromised patient. 2nd Edn. Chichester John Wiley and Sons, 1991:153-90.

53 Warnock DW, Johnson EM. Clinical manifestations and management of hyalohyphomycosis, phaeohyphomycosis and other uncommon forms of fungal infection in the and other uncommon . Wms of fungal infection in the eds. Fungal infection in the compromised patient. 2nd Edn. Chichester: John Wiley and Sons, 191:247-310.

54 Palfrey NA. Improved diagnosis and prognosis of mucormycosis. A clinicopathologic study of 33 cases. Medicine 1986;65:113-23.

55 Windus DW, Stokes TJ, Julian BA, Fenves AZ. Fatal Rhizopus infections in hemodialysis patients receiving deferoxamine. Ann Intern Med 1987;107:678-80.

56 Kaufman L, Turner LF, McLaughlin DW. Indirect enzyme-linked immunosorbent assay for zygomycosis. Clin Microbiol 1989;27:1979-82.

57 McGinnis MR, Rinaldi MG, Winn RE. Emerging agents of phaeohyphomycosis: pathogenic species of bipolaris and exserohilum. J Clin Microbiol 1986;24:250-9.

58 Richardson SE, Bannatyne RM, Summerbell RC, Milliken J, Gold R, Weitzman SS. Disseminated fusarial infection in the immunocompromised host. Rev Infect Dis in the immuno $10: 1171-81$.

59 Merz WG, Karp JE, Hoagland M, Jett-Goheen M, Junkins JM, Hood AF. Diagnosis and successful treatment of fusariosis in the compromised host. J Infect Dis 1988; 158: 1046-55.

60 Cheung LL, Hudson JB. Development of DNA probes for Candida albicans. Diagn Microbiol Infect Dis 1988;10:
171-9.

61 Spreadbury CL, Bainbridge BW, Cohen J. Restriction fragment length polymorphisms in isolates of Aspergillus fumigatus probed with part of the intergenic spacer region from the ribosomal gene complex of Aspergillus nidulans. J Gen Microbiol 1990;136:1991-4

62 Wakefield AE, Pixley FJ, Banerii S, et al. Detection of Pneumocystis carinii with DNA amplification. Lancet 1990;336:451-3.

63 Sugar AM. Empiric treatment of fungal infections in the neutropenic host: review of literature and guidelines for neutropenic host: review of literature and
use. Arch Intern Med 1990;150:2258-64.

64 Denning DW, Follansbee SE, Scolaro M, Norris S, Edelstein H, Stevens DA. Pulmonary aspergillosis in the acquired immunodeficiency syndrome. $N$ Engl in the 1991;324:654-62. 